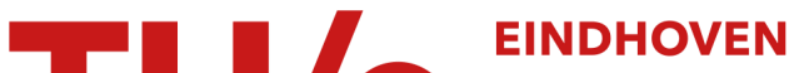 UNIVERSITY OF TECHNOLOGY
}

\section{Convergent piecewise affine systems : analysis and design Part I: continuous case}

\section{Citation for published version (APA):}

Pavlov, A. V., Wouw, van de, N., \& Nijmeijer, H. (2005). Convergent piecewise affine systems : analysis and design Part I: continuous case. In Proceedings of the 44th IEEE Conference on Decision and Control and European Control Conference(CDC-ECC), 12-15- december 2005, Seville, Spain (pp. on-CDROM). https://doi.org/10.1109/CDC.2005.1583019

DOI:

10.1109/CDC.2005.1583019

Document status and date:

Published: 01/01/2005

\section{Document Version:}

Accepted manuscript including changes made at the peer-review stage

\section{Please check the document version of this publication:}

- A submitted manuscript is the version of the article upon submission and before peer-review. There can be important differences between the submitted version and the official published version of record. People interested in the research are advised to contact the author for the final version of the publication, or visit the $\mathrm{DOI}$ to the publisher's website.

- The final author version and the galley proof are versions of the publication after peer review.

- The final published version features the final layout of the paper including the volume, issue and page numbers.

Link to publication

\section{General rights}

Copyright and moral rights for the publications made accessible in the public portal are retained by the authors and/or other copyright owners and it is a condition of accessing publications that users recognise and abide by the legal requirements associated with these rights.

- Users may download and print one copy of any publication from the public portal for the purpose of private study or research.

- You may not further distribute the material or use it for any profit-making activity or commercial gain

- You may freely distribute the URL identifying the publication in the public portal.

If the publication is distributed under the terms of Article 25fa of the Dutch Copyright Act, indicated by the "Taverne" license above, please follow below link for the End User Agreement:

www.tue.nl/taverne

Take down policy

If you believe that this document breaches copyright please contact us at:

openaccess@tue.nl

providing details and we will investigate your claim. 


\title{
Convergent piecewise affine systems: analysis and design Part I: continuous case
}

\author{
Alexey Pavlov, Nathan van de Wouw and Henk Nijmeijer
}

\begin{abstract}
In this paper convergence properties for piecewise affine (PWA) systems are studied. The notions of exponential, uniform and input-to-state convergence are introduced and studied. For PWA systems with continuous right-hand sides it is shown that the existence of a common quadratic Lyapunov function for the linear parts of the system dynamics in every mode is sufficient for the exponential and input-tostate convergence of the system. For a class of PWA control systems we design (output) feedback controllers that make the closed-loop system input-to-state convergent. The conditions for such controller design are formulated in terms of LMIs. The obtained results can be used for designing observers and (output-feedback) tracking controllers for PWA systems.
\end{abstract}

\section{INTRODUCTION}

In many control problems it is required that controllers are designed in such a way that all solutions of the corresponding closed-loop system "forget" their initial conditions. Actually, this is one of the main tasks of a feedback to eliminate dependency of solutions on initial conditions. In this case, all solutions converge to some steady-state solution which is determined only by the input of the closed-loop system. This input can be, for example, a command signal or a signal generated by a feedforward part of the controller or, as in the observer design problem, it can be the measured signal from the observed system. This convergence property of a system plays an important role in many (nonlinear) control problems including tracking, synchronization, observer design, the output regulation problem and performance analysis of nonlinear systems see e.g. [1], [2], [3], [4], [5] and references therein.

The property of convergence was formalized in the notion of convergent systems and studied first for periodically excited systems in [6] and then for systems with arbitrary excitations in [7], see also [8]. For systems in Lur'e form convergence was investigated in [9]. Similar properties have been studied in [10], [11]. Among recent papers one should mention the works [12], [13], [14], in which the authors studied convergence-like properties of dynamical systems using various formalizations, definitions and techniques.

In this paper we study the convergence properties for the class of piecewise affine (PWA) systems. This class

This research is partially supported by the Netherlands Organization for Scientific Research NWO and by the EU projects SICONOS(IST-200137172) and HyCon (FP6-2003-IST-2).

A. Pavlov is with Department of Engineering Cybernetics, Norwegian University of Science and Technology, NO-7491, Trondheim, Norway. N. van de Wouw and H. Nijmeijer are with Department of Mechanical Engineering, Eindhoven University of Technology, P.O.Box 513, $5600 \mathrm{MB}$ Eindhoven, The Netherlands. Alexey.Pavlovditk.ntnu.no, N.v.d.Wouw@tue.nl, H.Nijmeijer@tue.nl of systems attracted a lot of attention over the last years, see e.g. [15], [16], [17], [18]. This class includes mechanical systems with piecewise linear restoring characteristics, systems with friction, electrical circuits with diodes and other switching characteristics and control systems with switching controllers. In this paper we present conditions for convergence of PWA systems with continuous right-hand sides. The case of PWA systems with discontinuous righthand sides is considered in the second part of the paper [19]. Most of the known checkable conditions for convergence (or convergence-type properties like incremental stability, contraction, [7], [13], [14]) rely on linearization of the system and therefore they are not applicable to PWA systems, which are non-smooth systems. This fact indicates the novelty of the presented results. Moreover, based on the obtained conditions, we present a new result on observer design for PWA systems and a result on designing output-feedback controllers for PWA systems that make the corresponding closed-loop system convergent.

The paper is organized as follows. In Section II definitions of (uniformly, exponentially, input-to-state) convergent systems are given and some basic (interconnection) properties of convergent systems are presented. Sufficient conditions for the exponential and input-to-state convergence properties for PWA systems with continuous right-hand sides are provided in Section III. The problem of designing a controller for a PWA system that makes the corresponding closed-loop system convergent is addressed in Section IV. Section V contains conclusions.

\section{Convergent Systems}

In this section we give definitions of convergent systems. These definitions extend the definition given in [7]. Consider the system

$$
\dot{x}=f(x, t),
$$

where $x \in \mathbb{R}^{n}, t \in \mathbb{R}$ and $f(x, t)$ is locally Lipschitz in $x$ and piecewise continuous in $t$.

Definition 1 System (1) is said to be

- convergent if there exists a solution $\bar{x}(t)$ satisfying the following conditions

(i) $\bar{x}(t)$ is defined and bounded for all $t \in \mathbb{R}$,

(ii) $\bar{x}(t)$ is globally asymptotically stable.

- uniformly convergent if it is convergent and $\bar{x}(t)$ is globally uniformly asymptotically stable.

- exponentially convergent if it is convergent and $\bar{x}(t)$ is globally exponentially stable. 
The solution $\bar{x}(t)$ is called a steady-state solution. As follows from the definition of convergence, any solution of a convergent system "forgets" its initial condition and converges to some steady-state solution which is independent of the initial condition. In general, the steady-state solution $\bar{x}(t)$ may be non-unique. But for any two steady-state solutions $\bar{x}_{1}(t)$ and $\bar{x}_{2}(t)$ it holds that $\left|\bar{x}_{1}(t)-\bar{x}_{2}(t)\right| \rightarrow 0$ as $t \rightarrow+\infty$. At the same time, for uniformly convergent systems the steady-state solution is unique, as formulated below.

Property 1 ([4], [20]) If system (1) is uniformly convergent, then the steady-state solution $\bar{x}(t)$ is the only solution defined and bounded for all $t \in \mathbb{R}$.

Remark 1 In the original definition of convergent systems given in [7], the steady-state solution $\bar{x}(t)$ is required to be unique. In Definition 1 this requirement of uniqueness is omitted, since for the practically important case of uniform convergence uniqueness of the steady-state solution can be proved as a corollary to the definition of the uniform convergence.

The convergence property is an extension of stability properties of asymptotically stable linear time-invariant (LTI) systems. Recall that for a piecewise continuous vectorfunction $\phi(t)$, which is defined and bounded on $\mathbb{R}$, the system $\dot{x}=A x+\phi(t)$ with a Hurwitz matrix $A$ has a unique solution $\bar{x}(t)$ which is defined and bounded on $t \in(-\infty,+\infty)$. It is given by the formula $\bar{x}(t):=\int_{-\infty}^{t} \exp (A(t-s)) \phi(s) d s$. This solution is globally exponentially stable with the rate of convergence depending only on the matrix $A$. Thus, an asymptotically stable LTI system excited by a bounded piecewise-continuous function $\phi(t)$ is globally exponentially convergent.

In the scope of control problems, time dependency of the right-hand side of system (1) is usually due to some input. This input may represent, for example, a disturbance or a feedforward control signal. Below we will consider convergence properties for systems with inputs. So, instead of systems of the form (1), we consider systems

$$
\dot{x}=f(x, w)
$$

with state $x \in \mathbb{R}^{n}$ and input $w \in \mathbb{R}^{m}$. The function $f(x, w)$ is locally Lipschitz in $x$ and continuous in $w$. In the sequel we will consider the class $\overline{\mathbb{P C}}_{m}$ of piecewise continuous inputs $w(t): \mathbb{R} \rightarrow \mathbb{R}^{m}$ which are bounded for all $t \in \mathbb{R}$. Below we define the convergence property for systems with inputs.

Definition 2 System (2) is said to be (uniformly, exponentially) convergent if it is (uniformly, exponentially) convergent for every input $w \in \overline{\mathbb{P C}}_{m}$. In order to emphasize the dependency on the input $w(t)$, the steady-state solution is denoted by $\bar{x}_{w}(t)$.

The next statement summarizes some properties of uniformly convergent systems excited by periodic or constant inputs.
These properties are natural for linear systems, whereas for nonlinear systems they, in general, do not hold.

Property 2 ([7]) Suppose system (2) with a given input $w(t)$ is uniformly convergent. If the input $w(t)$ is constant, the corresponding steady-state solution $\bar{x}_{w}(t)$ is also constant; if the input $w(t)$ is periodic with period $T$, then the corresponding steady-state solution $\bar{x}_{w}(t)$ is also periodic with the same period $T$.

The next definition extends the uniform convergence property to the input-to-state stability framework.

Definition 3 System (2) is said to be input-to-state convergent if it is globally uniformly convergent and for every input $w \in \overline{\mathbb{P C}}_{m}$ system (2) is ISS with respect to the steady-state solution $\bar{x}_{w}(t)$, i.e. there exist a $\mathcal{K} \mathcal{L}$-function $\beta(r, s)$ and a $\mathcal{K}_{\infty}$-function $\gamma(r)$ such that any solution $x(t)$ of system (2) corresponding to some input $\hat{w}(t):=w(t)+\Delta w(t)$ satisfies

$$
\begin{array}{r}
\left|x(t)-\bar{x}_{w}(t)\right| \leq \beta\left(\left|x\left(t_{0}\right)-\bar{x}_{w}\left(t_{0}\right)\right|, t-t_{0}\right) \\
+\gamma\left(\sup _{t_{0} \leq \tau \leq t}|\Delta w(\tau)|\right) .
\end{array}
$$

In general, the functions $\beta(r, s)$ and $\gamma(r)$ may depend on the particular input $w(t)$. If $\beta(r, s)$ and $\gamma(r)$ are independent of the input $w(t)$, then such system is called uniformly inputto-state convergent.

Similar to the conventional ISS property, the property of input-to-state convergence is especially useful for studying convergence properties of interconnected systems. One can easily show that parallel interconnection of (exponentially, uniformly, input-to-state) convergent systems is again an (exponentially, uniformly, input-to-state) convergent system. Series connection of two input-to-state convergent systems is an input-to-state convergent system, as summarized in the next property.

\section{Property 3 ([4], [20]) Consider the system}

$$
\left\{\begin{aligned}
\dot{x} & =f(x, y, w), & & x \in \mathbb{R}^{n} \\
\dot{y} & =g(y, w), & & y \in \mathbb{R}^{q} .
\end{aligned}\right.
$$

Suppose the $x$-subsystem with $(y, w)$ as input is input-tostate convergent and the $y$-subsystem with $w$ as input is input-to-state convergent. Then system (4) is input-to-state convergent.

The next property deals with bidirectionally interconnected input-to-state convergent systems.

Property 4 ([4], [20]) Consider the system

$$
\begin{cases}\dot{x}=f(x, y, w), & x \in \mathbb{R}^{n} \\ \dot{y}=g(x, y, w), & y \in \mathbb{R}^{q} .\end{cases}
$$

Suppose the $x$-subsystem with $(y, w)$ as inputs is inputto-state convergent. Assume that there exists a class $\mathcal{K} \mathcal{L}$ function $\beta_{y}(r, s)$ such that for any input $(x, w) \in \overline{\mathbb{P C}}_{n+m}$ any solution of the $y$-subsystem satisfies

$$
|y(t)| \leq \beta_{y}\left(\left|y\left(t_{0}\right)\right|, t-t_{0}\right) .
$$


Then the interconnected system (5) is input-to-state convergent.

Remark. Property 4 can be used for establishing the separation principle for input-to-state convergent systems as it will be done in Section IV. In that context system (5) represents a system in closed loop with a state-feedback controller and an observer generating state estimates for this controller. The $y$-subsystem corresponds to the observer error dynamics.

Notice that the (uniform) convergence and the inputto-state convergence properties are invariant under smooth coordinate transformations, since all the ingredients in the definitions of these properties (see Definitions 1-3) are invariant under smooth coordinate transformations.

\section{CONVERGENT PIECEWISE AFFINE SYSTEMS}

In the previous sections we presented the definitions and basic properties of convergent systems. The next question to be addressed is: how to check whether a system exhibits these convergence properties? For smooth systems this question has been answered in [7], whereas for non-smooth systems this question has been answered only for systems in Lur'e form with one (non-smooth) scalar nonlinearity, see [9]. Piecewise affine systems constitute an important class of non-smooth systems. In this section we provide sufficient conditions for convergence of piecewise-affine systems with continuous right-hand sides.

Consider the state space $\mathbb{R}^{n}$ divided into polyhedral cells $\Lambda_{i}, i=1, \ldots, l$, by hyperplanes given by equations of the form $H_{j}^{T} z+h_{j}=0$, for some $H_{j} \in \mathbb{R}^{n}$ and $h_{j} \in \mathbb{R}$, $j=1, \ldots, k$. We will consider piecewise-affine systems of the form

$$
\dot{x}=A_{i} x+b_{i}+D w, \quad \text { for } x \in \Lambda_{i}, i=1, \ldots, l .
$$

Here $A_{i} \in \mathbb{R}^{n \times n}$ and $b_{i} \in \mathbb{R}^{n}, i=1, \ldots, l$, are constant matrices and vectors, respectively. The vector $x \in \mathbb{R}^{n}$ is the state and $w \in \mathbb{R}^{m}$ is the input. The hyperplanes $H_{j}^{T} x+$ $h_{j}=0, j=1, \ldots, k$, are the switching surfaces. In the sequel we will deal with piecewise affine systems which have continuous right-hand sides. This continuity requirement on the right-hand side of system (6) can be characterized by the following simple algebraic lemma. Its proof can be found, for example, in [4].

Lemma 1 Consider system (6). The right-hand side of system (6) is continuous iff the following condition is satisfied: for any two cells $\Lambda_{i}$ and $\Lambda_{j}$ having a common boundary $H^{T} z+h=0$ the corresponding matrices $A_{i}$ and $A_{j}$ and the vectors $b_{i}$ and $b_{j}$ satisfy the equalities

$$
\begin{aligned}
G_{H} H^{T} & =A_{i}-A_{j} \\
G_{H} h & =b_{i}-b_{j},
\end{aligned}
$$

for some vector $G_{H} \in \mathbb{R}^{n}$.

The following theorem establishes sufficient conditions for the exponential and input-to-state convergence of system (6).
Theorem 1 Consider system (6). Suppose the right-hand side of (6) is continuous and there exists a positive definite matrix $P=P^{T}>0$ such that

$$
P A_{i}+A_{i}^{T} P<0, \quad i=1, \ldots, l .
$$

Then system (2) is exponentially convergent and input-tostate convergent.

Before giving the proof of this theorem, we formulate and prove an important technical lemma, which will be used in the proof of the theorem and in further analysis in Section IV. Denote the right-hand side of (6) $f(x, w)$.

Lemma 2 Under the conditions of Theorem 1 it holds that

$$
\begin{array}{r}
\left(x_{1}-x_{2}\right)^{T} P\left(f\left(x_{1}, w\right)-f\left(x_{2}, w\right)\right) \\
\leq-\alpha\left(x_{1}-x_{2}\right)^{T} P\left(x_{1}-x_{2}\right) .
\end{array}
$$

for all $x_{1}, x_{2} \in \mathbb{R}^{n}, w \in \mathbb{R}^{m}$, for some $\alpha>0$ and for the matrix $P$ satisfying (8).

Proof: Since $P$ satisfies LMI (8), there exists a constant $\alpha>0$ such that

$$
P A_{i}+A_{i}^{T} P \leq-2 \alpha P, \quad i=1, \ldots, l .
$$

Let us show that this $\alpha$ is the constant for which inequality (9) holds for all $x_{1}, x_{2} \in \mathbb{R}^{n}$ and all $w \in \mathbb{R}^{m}$. We will show this in two steps. First, consider the case when both $x_{1}$ and $x_{2}$ belong to the same cell $\Lambda_{i}$ with the dynamics $\dot{x}=A_{i} x+b_{i}+D w$. Then, $f\left(x_{1}, w\right)=A_{i} x_{1}+b_{i}+D w$ and $f\left(x_{2}, w\right)=A_{i} x_{2}+b_{i}+D w$. Therefore,

$$
\begin{aligned}
& \left(x_{1}-x_{2}\right)^{T} P\left(f\left(x_{1}, w\right)-f\left(x_{2}, w\right)\right) \\
& =\left(x_{1}-x_{2}\right)^{T} P\left(A_{i} x_{1}-A_{i} x_{2}\right) \\
& =\frac{1}{2}\left(x_{1}-x_{2}\right)^{T}\left(P A_{i}+A_{i}^{T} P\right)\left(x_{1}-x_{2}\right) \\
& \leq-\alpha\left(x_{1}-x_{2}\right)^{T} P\left(x_{1}-x_{2}\right) .
\end{aligned}
$$

Thus, inequality (9) holds for any pair of points $x_{1}$ and $x_{2}$ lying in the same cell $\Lambda_{i}$.

Next, we consider the case of arbitrary $x_{1}$ and $x_{2}$. Consider the line segment $\left[x_{1}, x_{2}\right]$ connecting these two points.

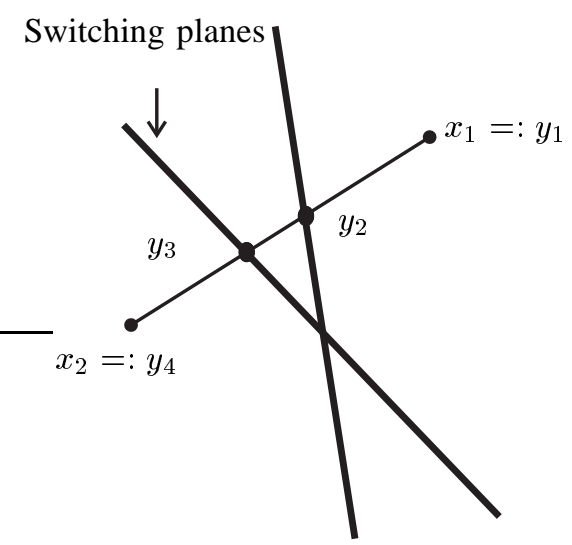

Fig. 1. The line segment $\left(x_{1}, x_{2}\right)$ intersects the switching planes in the points $y_{1}, \ldots, y_{4}$. 
Denote $y_{1}:=x_{1}, y_{p}:=x_{2}$ and $y_{i}, i=2, \ldots, p-1$, - the points of intersection of the line segment $\left[x_{1}, x_{2}\right]$ with the switching surfaces such that any pair of points $y_{i}$, $y_{i}+1$ belongs to the same cell $\Lambda_{j}$ (including its borders), $y_{i} \neq y_{i+1}, i=1, \ldots, p-1$, and the sequence $y_{1}, y_{2}, \ldots, y_{p}$ is ordered, see Fig. 1 Denote $e:=\left(x_{1}-x_{2}\right) /\left|x_{1}-x_{2}\right|_{P}$, where $|x|_{P}:=\sqrt{x^{T} P x}$. Since all points $y_{i}, i=1, \ldots, p$, lie on the same line segment $\left[x_{1}, x_{2}\right]$ and they are ordered, then

$$
e=\frac{y_{i}-y_{i+1}}{\left|y_{i}-y_{i+1}\right|_{P}}, \quad i=1, \ldots, p-1 .
$$

Taking this fact into account, we obtain

$$
\begin{aligned}
& \left(x_{1}-x_{2}\right)^{T} P\left(f\left(x_{1}, w\right)-f\left(x_{2}, w\right)\right) \\
& =\left|x_{1}-x_{2}\right|_{P} \sum_{i=1}^{p-1} e^{T} P\left(f\left(y_{i}, w\right)-f\left(y_{i+1}, w\right)\right) \\
& =\left|x_{1}-x_{2}\right|_{P} \times \\
& \times \sum_{i=1}^{p-1} \frac{\left(y_{i}-y_{i+1}\right)^{T} P\left(f\left(y_{i}, w\right)-f\left(y_{i+1}, w\right)\right)}{\left|y_{i}-y_{i+1}\right|_{P}} .
\end{aligned}
$$

Since each pair of points $y_{i}$ and $y_{i+1}, i=1, \ldots, p-1$, belongs to a cell with the same dynamics, from the first step of the proof we obtain

$$
\begin{gathered}
\left(y_{i}-y_{i+1}\right)^{T} P\left(f\left(y_{i}, w\right)-f\left(y_{i+1}, w\right)\right) \\
\leq-\alpha\left(y_{i}-y_{i+1}\right)^{T} P\left(y_{i}-y_{i+1}\right) .
\end{gathered}
$$

Substituting this inequality into (13), implies

$$
\begin{aligned}
& \left(x_{1}-x_{2}\right)^{T} P\left(f\left(x_{1}, w\right)-f\left(x_{2}, w\right)\right) \\
& \leq-\alpha\left|x_{1}-x_{2}\right|_{P} \sum_{i=1}^{p-1}\left|y_{i}-y_{i+1}\right|_{P} .
\end{aligned}
$$

Since all points $y_{i}, i=1, \ldots, p$, lie on the same line segment $\left[x_{1}, x_{2}\right]$ and they are ordered,

$$
\sum_{i=1}^{p-1}\left|y_{i}-y_{i+1}\right|_{P}=\left|y_{1}-y_{p}\right|_{P}=\left|x_{1}-x_{2}\right|_{P} .
$$

This fact together with (15) implies (9). Due to the arbitrary choice of $x_{1}, x_{2}$ and $w$ we obtain that (9) holds for all $w \in \mathbb{R}^{m}$ and all $x_{1}, x_{2} \in \mathbb{R}^{n}$. This completes the proof of this lemma.

Now we can prove Theorem 1.

Proof: Given the result of Lemma 2, the proof of exponential convergence repeats the proof from [21], [8]. We only need to show that system (6) is input-to-state convergent. Consider some input $w(t)$ and the corresponding steady-state solution $\bar{x}_{w}(t)$. Let $x(t)$ be a solution of system (6) corresponding to some input $\hat{w}(t)$. Denote $\Delta x:=x-\bar{x}_{w}(t)$ and $\Delta w:=$ $\hat{w}-w(t)$. Then $\Delta x$ satisfies the equation

$$
\Delta \dot{x}=f\left(\bar{x}_{w}(t)+\Delta x, w(t)+\Delta w\right)-f\left(\bar{x}_{w}(t), w(t)\right),
$$

where $f(x, w)$ denotes the right-hand side of system (6). We will show that system (17) with $\Delta w$ as input is ISS. Due to the arbitrary choice of $w(t)$, this fact implies that system (6) is input-to-state convergent.
Consider the function $V(\Delta x)=\frac{1}{2}(\Delta x)^{T} P \Delta x$. Its derivative along solutions of system (17) satisfies $\dot{V}=$

$$
\begin{gathered}
\Delta x^{T} P\left\{f\left(\bar{x}_{w}(t)+\Delta x, w(t)+\Delta w(t)\right)-f\left(\bar{x}_{w}(t), w(t)\right)\right\} \\
=\Delta x^{T} P\left\{f\left(\bar{x}_{w}(t)+\Delta x, w(t)+\Delta w(t)\right)\right. \\
\left.-f\left(\bar{x}_{w}(t), w(t)+\Delta w(t)\right)\right\} \\
+\Delta x^{T} P\left\{f\left(\bar{x}_{w}(t), w(t)+\Delta w(t)\right)-f\left(\bar{x}_{w}(t), w(t)\right)\right\}
\end{gathered}
$$

Applying Lemma 2 to the first component in (18), we obtain

$$
\begin{aligned}
& \Delta x^{T} P\left\{f\left(\bar{x}_{w}(t)+\Delta x, w(t)+\Delta w(t)\right)\right. \\
& \left.-f\left(\bar{x}_{w}(t), w(t)+\Delta w(t)\right)\right\} \leq-\alpha|\Delta x|_{P}^{2},
\end{aligned}
$$

where $|\Delta x|_{P}^{2}:=(\Delta x)^{T} P \Delta x$. Since $f(x, w)$, the right-hand side of system (6), is linear in $w$, the second component in formula (18) equals

$$
\begin{array}{r}
\Delta x^{T} P\left\{f\left(\bar{x}_{w}(t), w(t)+\Delta w(t)\right)-\right. \\
\left.f\left(\bar{x}_{w}(t), w(t)\right)\right\} \\
=\Delta x^{T} P D \Delta w .
\end{array}
$$

Applying the Cauchy inequality to (20), we obtain

$$
\left|\Delta x^{T} P D \Delta w\right| \leq|\Delta x|_{P}|D \Delta w|_{P} \leq c|\Delta x|_{P}|\Delta w|
$$

where the constant $c$ depends only on $D$ and $P$. After substituting this estimate together with estimates (19) and (20) in formula (18), we obtain

$$
\frac{d V}{d t} \leq-\alpha|\Delta x|_{P}^{2}+|\Delta x|_{P} c|\Delta w|
$$

From this formula we obtain

$$
\frac{d V}{d t} \leq-\frac{\alpha}{2}|\Delta x|_{P}^{2}, \quad \forall|\Delta x|_{P} \geq \frac{2 c}{\alpha}|\Delta w| .
$$

By the Lyapunov characterization of the ISS property (see e.g. [22], Theorem 5.2), we obtain that system (17) is inputto-state stable. This completes the proof of the theorem.

Theorem 1 not only allows to check the input-to-state convergence property for a given system, but also serves as a useful tool in designing controllers that make the corresponding closed-loop system convergent. This controller design problem is considered in Section IV.

From the result of Theorem 1 one may conjecture that for a PWA system with a discontinuous right-hand side, the existence of a common quadratic Lyapunov function for the linear parts of the dynamics in all modes is also sufficient for convergence. Yet, this conjecture is not true, as follows from a counterexample presented in the second part of this paper [19]. In [19] we study convergent PWA systems with discontinuous right-hand sides.

\section{Controller Design For CONVERGENT Systems}

The convergence property is desirable in many control problems because the steady-state dynamics of a convergent system are independent of the initial conditions. In this section we address the problem of how to achieve the 
convergence property in a piecewise affine control system by means of feedback. Consider the following PWA system

$$
\begin{aligned}
\dot{x} & =A_{i} x+b_{i}+B u+D w, \text { for } x \in \Lambda_{i}, i=1, \ldots, l \\
y & =C x+E w
\end{aligned}
$$

with state $x \in \mathbb{R}^{n}$, control $u \in \mathbb{R}^{k}$, external input $w \in \mathbb{R}^{m}$ and output $y \in \mathbb{R}^{p}$. Here $A_{i}, b_{i}, i=1, \ldots, l, B, D, C$ and $E$ are constant matrices of the appropriate dimensions. As in the previous section, $\Lambda_{i}$ are polyhedral cells with disjoint interior which together constitute the state space $\mathbb{R}^{n}$. In this setting the input $u$ corresponds to the feedback part of the controller. The input $w$ includes external timedependent inputs such as, for example, disturbances and feedforward control signals. Once the convergence property is achieved by a proper choice of feedback, the feedforward control signals can be used in order to shape the steady-state dynamics of the closed-loop system (see e.g. [4], [23]). We will focus on the problem of finding a feedback that makes the closed-loop system input-to-state convergent and will not address the problem of shaping the steady-state dynamics by means of a feedforward controller.

The following lemma provides conditions under which there exists a state feedback rendering the corresponding closed-loop system input-to-state convergent.

Lemma 3 Consider the system (24). Suppose the right-hand side of (24) is continuous and the LMI

$$
\begin{aligned}
\mathcal{P}_{c}=\mathcal{P}_{c}^{T} & >0, \\
A_{i} \mathcal{P}_{c}+\mathcal{P}_{c} A_{i}^{T}+B \mathcal{Y}+\mathcal{Y}^{T} B^{T} & <0, i=1, \ldots, l,
\end{aligned}
$$

is feasible. Then the system (24) in closed-loop with the controller $u=K(x+v)$ with $K:=\mathcal{Y}_{\mathcal{P}_{c}^{-1}}$ and $(v, w)$ as inputs is input-to-state convergent.

Proof: The closed-loop system has the form

$$
\dot{x}=\left(A_{i}+B K\right) x+b_{i}+B K v+D w, \quad x \in \Lambda_{i},
$$

$i=1, \ldots, l$. Since the right-hand side of system (24) is continuous, the right-hand side of the closed-loop system (26) is also continuous. Since the LMI (25) is feasible, for the matrix $K:=\mathcal{Y} \mathcal{P}_{c}^{-1}$ it holds that

$$
\mathcal{P}_{c}^{-1}\left(A_{i}+B K\right)+\left(A_{i}+B K\right)^{T} \mathcal{P}_{c}^{-1}<0, \quad i=1, \ldots, l .
$$

Therefore, the closed-loop system (26) satisfies the conditions of Theorem 1 with the matrix $P:=\mathcal{P}_{c}^{-1}>0$. Hence, system (26) with $(v, w)$ as inputs is input-to-state convergent.

The next lemma shows how to design an observer based on the convergence property. Here we assume that the external signal $w(t)$ is measured.

Lemma 4 Consider system (24). Suppose the right-hand side of (24) is continuous and the LMI

$$
\begin{aligned}
\mathcal{P}_{o}=\mathcal{P}_{o}^{T} & >0 \\
\mathcal{P}_{o} A_{i}+A_{i}^{T} \mathcal{P}_{o}+\mathcal{X} C+C^{T} \mathcal{X}^{T} & <0, i=1, \ldots l
\end{aligned}
$$

is feasible. Then the system

$$
\begin{aligned}
\dot{\hat{x}} & =A_{i} \hat{x}+b_{i}+B u+D w+L(\hat{y}-y), \quad \hat{x} \in \Lambda_{i}, \\
\hat{y} & =C \hat{x}+E w, \quad i=1, \ldots, l,
\end{aligned}
$$

with $L:=\mathcal{P}_{o}^{-1} \mathcal{X}$, is an observer for system (24) with globally exponentially stable error dynamics. Moreover, the observer error dynamics

$$
\Delta \dot{x}=g(x+\Delta x, u, w)-g(x, u, w),
$$

where $g(x, u, w):=A_{i} x+b_{i}+B u+D w+L(C x+E w)$ for $x \in \Lambda_{i}, i=1, \ldots, l$, is such that for any bounded $x(t)$ and $w(t)$ and any feedback $u=U(\Delta x, t)$ all solutions of system (29) satisfy

$$
|\Delta x(t)| \leq c e^{-a\left(t-t_{0}\right)}\left|\Delta x\left(t_{0}\right)\right|,
$$

where the numbers $c>0$ and $a>0$ are independent of $x(t)$, $w(t)$ and $u=U(\Delta x, t)$.

Proof: Let us first prove the second part of the lemma. Consider the function $g(x, u, w)$. After unifying the terms containing $x$, we obtain $g(x, u, w):=\left(A_{i}+L C\right) x+b_{i}+$ $B u+(D+L E) w$ for $x \in \Lambda_{i}, i=1, \ldots, l$. Since the righthand side of system (24) is continuous, then $g(x, u, w)$ is also a continuous piecewise-affine function. Moreover, since the LMI (27) is feasible, for $P:=\mathcal{P}_{o}$ and $L:=\mathcal{P}_{o}^{-1} \mathcal{X}$ it holds that

$$
P\left(A_{i}+L C\right)+\left(A_{i}+L C\right)^{T} P<0, \quad i=1, \ldots, l .
$$

Applying Lemma 2 to the function $g(x, u, w)$, we obtain

$$
\Delta x^{T} P(g(x+\Delta x, u, w)-g(x, u, w)) \leq-a \Delta x^{T} P \Delta x
$$

for all $x, \Delta x, u$ and $w$ and some constant $a>0$ independent of $x, \Delta x, u$ and $w$. Consider the function $V(\Delta x):=$ $1 / 2 \Delta x^{T} P \Delta x$. The derivative of this function along solutions of system (29) satisfies

$\frac{d V}{d t}=\Delta x^{T} P(g(x+\Delta x, u, w)-g(x, u, w)) \leq-2 a V(\Delta x)$.

This inequality, in turn, implies that there exists $c>0$ depending only on the matrix $P$ such that if $x(t)$ and $w(t)$ are defined for all $t \geq t_{0}$ then the solution $\Delta x(t)$ is also defined for all $t \geq t_{0}$ and satisfies (30). It remains to show that system (28) is an observer for system (24). Denote $\Delta x:=\hat{x}-x(t)$. Since $x(t)$ is a solution of system (24), $\Delta x(t)$ satisfies equation (29). By the previous analysis, we obtain that $\Delta x(t)$ satisfies (30). Therefore, the observation error $\Delta x(t)$ exponentially tends to zero.

Lemmas 3 and 4 show how to design a state feedback controller that makes the closed-loop system input-to-state convergent and how to design an observer for this system with an exponentially stable error dynamics. In fact, for such controllers and observers one can use the separation principle in order to design an output feedback controller that makes the closed-loop system input-to-state convergent. This statement follows from the next theorem. 
Theorem 2 Consider the system (24). Suppose the LMIs (25) and (27) are feasible. Denote $K:=\mathcal{Y P}_{c}^{-1}$ and $L:=$ $\mathcal{P}_{o}^{-1} \mathcal{X}$. Then system (24) in closed loop with the controller

$$
\begin{aligned}
& \dot{\hat{x}}=A_{i} \hat{x}+b_{i}+B u+D w+L(\hat{y}-y), \quad \hat{x} \in \Lambda_{i}, \\
& u=K \hat{x} \quad i=1, \ldots, l,
\end{aligned}
$$

with $w$ as an input is input-to-state convergent.

Proof: Denote $\Delta x:=\hat{x}-x$. Then in the new coordinates $(x, \Delta x)$ the equations of the closed-loop system are

$$
\begin{aligned}
\dot{x} & =\left(A_{i}+B K\right) x+b_{i}+B K \Delta x+D w, x \in \Lambda_{i} \\
\Delta \dot{x} & =g(x+\Delta x, u, w)-g(x, u, w) \\
u & =K(x+\Delta x)
\end{aligned}
$$

By the choice of $K$, system (33) with $(\Delta x, w)$ as inputs is input-to-state convergent (see Lemma 3). By the choice of the observer gain $L$, for any inputs $x(t), w(t)$ and for the feedback $u=K(x(t)+\Delta x)$, any solution of system (34), (35) satisfies

$$
|\Delta x(t)| \leq c e^{-a\left(t-t_{0}\right)}\left|\Delta x\left(t_{0}\right)\right|,
$$

where the numbers $c>0$ and $a>0$ are independent of $x(t)$ and $w(t)$ (see Lemma 4). Applying Property 4, we obtain that the closed-loop system (33)-(35) is input-to-state convergent.

\section{Conclusions}

In this paper we have studied convergence properties for piecewise affine (PWA) systems. We have introduced the notions of exponential, uniform and input-to-state convergence and studied their basic (interconnection) properties. For PWA systems with continuous right-hand sides it has been shown that the existence of a common quadratic Lyapunov function for the linear parts of the system dynamics in every mode is sufficient for the exponential and input-to-state convergence of the system. Based on this result, for a class of PWA control systems we have designed observers and (output) feedback controllers that make the closed-loop system input-to-state convergent. The conditions for such observer and controller design are formulated in terms of LMIs. The obtained results can be used for designing observers and (output-feedback) tracking controllers for PWA systems.

\section{REFERENCES}

[1] A. Pogromsky, "Passivity based design of synchronizing systems," In. J. Bifurcation Chaos, vol. 8(2), pp. 295-319, 1998.

[2] A. Juloski, M. Heemels, and S. Weiland, "Observer design for a class of piecewise affine systems," in Proc. of IEEE Conf. Decision and Control, 2002.

[3] A. Pavlov, N. van de Wouw, and H. Nijmeijer, "The uniform global output regulation problem," in Proc. of IEEE Conf. Decision and Control, 2004.

[4] A. Pavlov, "The output regulation problem: a convergent dynamics approach," Ph.D. dissertation, Eindhoven University of Technology, Eindhoven, 2004.

[5] M. Heertjes and M. Steinbuch, "Stability and performance of variable gain controllers with application to a DVD storage drive," Automatica, vol. 40, pp. 591-602, 2004.
[6] V. Pliss, Nonlocal problems of the theory of oscillations. London: Academic Press, 1966.

[7] B. Demidovich, Lectures on stability theory (in Russian). Moscow: Nauka, 1967.

[8] A. Pavlov, A. Pogromsky, N. van de Wouw, and H. Nijmeijer, "Convergent dynamics, a tribute to Boris Pavlovich Demidovich," Systems and Control Letters, vol. 52, pp. 257-261, 2004.

[9] V. Yakubovich, "Matrix inequalities method in stability theory for nonlinear control systems: I. absolute stability of forced vibrations," Automation and Remote Control, vol. 7, pp. 905-917, 1964.

[10] J. LaSalle and S. Lefschetz, Stability by Liapunov's direct method with applications. New York: Academic press, 1961.

[11] T. Yoshizawa, Stability theory by Liapunov's second method. Tokio: The Mathematical Society of Japan, 1966.

[12] D. Angeli, "A Lyapunov approach to incremental stability properties," IEEE Trans. Automatic Control, vol. 47, pp. 410-421, 2002.

[13] V. Fromion, G. Scorletti, and G. Ferreres, "Nonlinear performance of a PI controlled missile: an explanation," Int. J. Robust Nonlinear Control, vol. 9, pp. 485-518, 1999.

[14] W. Lohmiller and J.-J. Slotine, "On contraction analysis for nonlinear systems," Automatica, vol. 34, pp. 683-696, 1998.

[15] M. Johansson and A. Rantzer, "Computation of piecewise quadratic lyapunov functions for hybrid systems," IEEE Trans. Automatic Control, vol. 43, pp. 555-559, 1998.

[16] W. Heemels, M. Camlibel, and J. Schumacher, "On the dynamic analysis of piecewise-linear networks," IEEE Trans. Circuits and Systems I: Fundamental Theory and Applications, vol. 49, pp. 315$327,2002$.

[17] A. Bemporad, G. Ferrari-Trecate, and M. Morari, "Observability and controllability of piecewise affine and hybrid systems," IEEE Trans. Automatic Control, vol. 45, pp. 1864-1876, 2000.

[18] D. Liberzon, Switchings in systems and control. Boston: Birkhauser 2003.

[19] A. Pavlov, A. Pogromsky, N. van de Wouw, and H. Nijmeijer, "Convergent piecewise affine systems: analysis and design. Part II: discontinuous case." in Proc. of IEEE Conf. Decision and Control, 2005.

[20] A. Pavlov, N. van de Wouw, and H. Nijmeijer, "Convergent systems: analysis and design," in Symposium on Nonlinear Controller and Observer Design - From Theory to Applications, 2005.

[21] B. Demidovich, "Dissipativity of a nonlinear system of differential equations, part I," Vestnik Moscow State Univiersity, ser. matem. mekh., (in Russian), vol. 6, pp. 19-27, 1961.

[22] H. Khalil, Nonlinear systems, 2nd ed. Upper Saddle River: Prentice Hall, 1996.

[23] A. Pavlov, N. van de Wouw, and H. Nijmeijer, "The global output regulation problem: an incremental stability approach," in Proc. of 6th IFAC Symposium on Nonlinear Control Systems, 2004. 